\title{
Relationship between Emotional Intelligence, Emotion Regulation and Emotional Expressivity in Employees
}

\author{
ASMA RASHID \\ Department of Psychology, University of Sargodha. \\ Email: asma.rashid3344@gmail.com \\ Tel: +923215074209 \\ MASUD AKHTAR \\ Department of Psychology, University of Lahore. \\ Email: masud.akhtar@sgd.uol.edu.pk \\ Tel: +923071112294 \\ MUHAMMAD NA VEED RIAZ \\ Department of Psychology, University of Sargodha. \\ Email: naveed.riaz@uos.edu.pk \\ Tel: +923007714217
}

\begin{abstract}
The study examined the relationship between emotional intelligence, emotional regulation and emotional expressivity in employees. Present study was descriptive research based on survey research design conducted in various offices situated in Sargodha. Data from A purposive sample of employees $(N=200)$ was collected using three scales. Results revealed significant relationship between emotional intelligence, emotional regulation and emotional expressivity in employees.: The finding has theoretical and applied significant in term of relationship between three interrelated constructs based on management of emotions at workplace.
\end{abstract}

Keywords: Emotional Expressivity, Emotional Regulation, Emotional Intelligence, Employees.

\section{Introduction}

Over the last few decades, technical innovation has resulted in a major change in how people interact. Although face-to-face contact used to be the norm of conversation, we now live in a period of rapid transition. Modern interlocutors may exchange fast and effective computer-mediated communications due to the widespread availability of portable electronic devices. Emoticons are visual symbols that use punctuation marks and letters to depict facial expressions in order to communicate a person's emotion, add meaning to textual communication, and explain a message that may otherwise be misconstrued (Lyons, 2018). According to study, embryonic symbols emerge as cartoon-like images and perform a number of essential functions: emoticons provide a positive effect, affect perception management, and complement nonverbal communication (Cardinosa-Queriones, Alvaro, \& Rosete, 2018; Vandergriff, 2013).

Emotional intelligence is the ability of a person to understand his or her own emotions, others' emotions and to better adjust in the changing environments. More specifically, the study has focused the Process Model of Emotion Régulation by Gross (2014) which illustrates a multi-stage process of emotion regulation. Emotional expressivity is based on the ability of the individual to make use of verbal and non- 
verbal expression of both positive and negative emotions in daily life scenarios (Riggio, 2017). Past literature confirmed that these three constructs are correlated with each other.

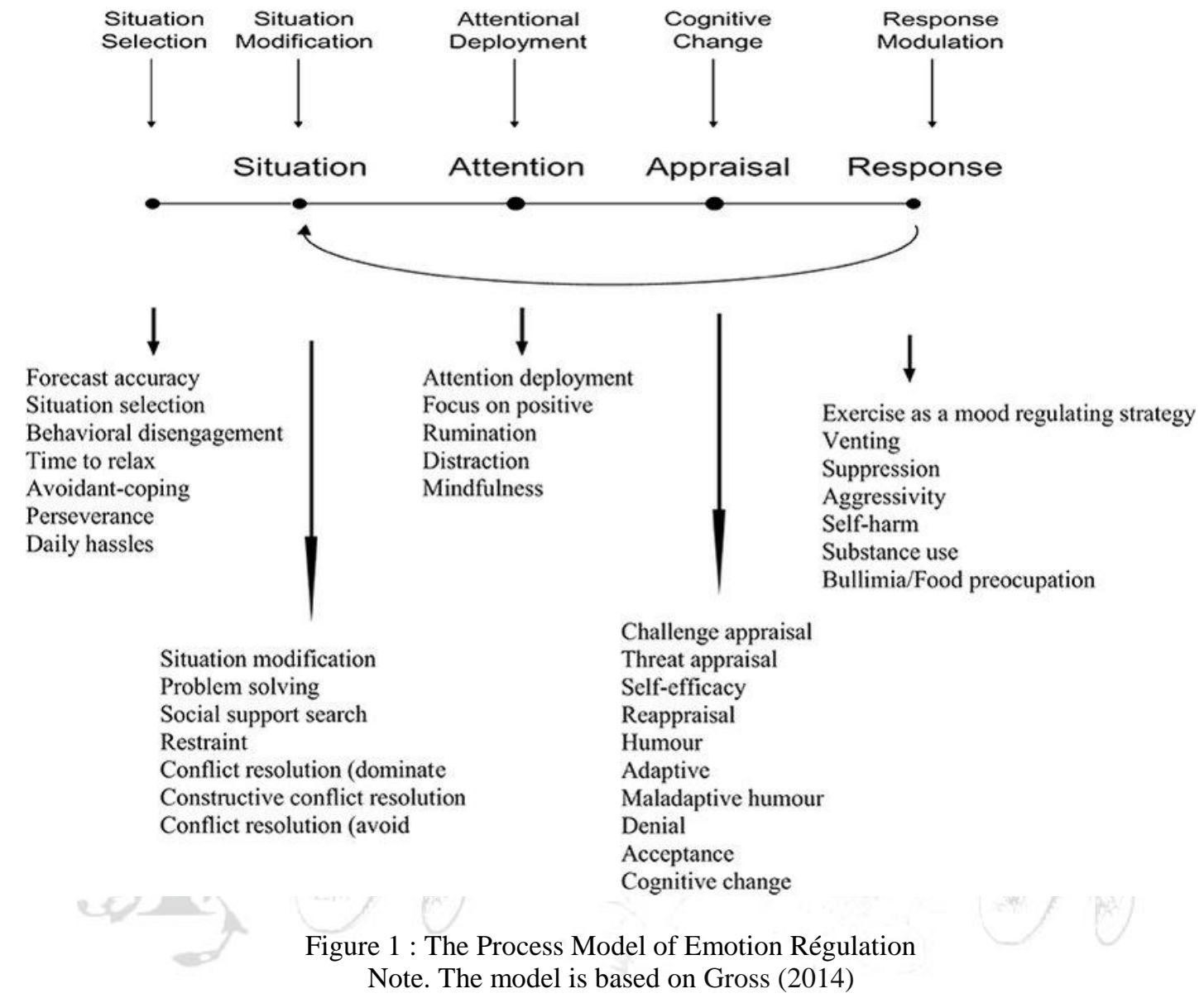

\section{Literature Review}

Very few has been carried out to determine the relationship between these concepts/variables. So, the present research will be a valuable addition in the literature history. Although modern research has focused on the integration of emotional intelliegence, emotion regulation and emotional expressivity (PeñaSarrionandia, Mikolajczak, \& Gross, 2019; Wojciechowski, Stolarski, \& Matthews, 2014). The study conducted in west on variables like emotion regulation and emotional expression (Brandão et al., 2016; Manalai et al., 2012), emotional well-being (Zhang, \& Zheng, 2017; Lin, \& Qiu, 2012), emoticons (emoji) and emotions (Kim, \& Park, 2018; Sarkar, Shetty, \& Humstoe, 2014). In Pakistan, the study was conducted on emoticons in computer-mediated communication (Jibril, \& Abdullah, 2013) but they lack the psychological element of using these sideways emotions.

This study is unique in a way that combination of the variables is different from previous studies. In the indigenous content there was not such literary history that can identify the rate of emotions in the prediction of emotions expressivity, emotional intelligence, and emotional regulation among students in Pakistan so the present research will be valuable study to study the role of the sideway emotions among surdents of Pakistan. 


\section{Methodology}

Present study was descriptive research. Participants were employees of different offices $(N=200)$ situated in Sargodha which were approached using purposive sampling technique age ranged from 25-55 years. Following three scales were administered on employees to collect data. The inclusion criteria was based on "regular job" and "one year experience".

Emotional Expressivity Questionnaire was constructed to measure the emotional expression of a person. This scale was developed by Gross and John (1997). It consists of 16 items with three subscales including negative expressivity, positive expressivity, and impulse strength. The scale is based on a 7-point Likert scale response format. Maximum and minimum scores are ranged from 16 to 80. High scores represent high emotional expressivity and low scores show low sense of emotional expressivity. The items numbered 3,8 , and 9 are reverse scored while other items are positively worded. The reliability of the original scale was determined through alpha that was .86. Past research in European context pointed out it was reliable and valid to quantify emotional expressivity (Gross \& John, 1997).

Emotional Regulation Questionnaire was designed to measure the ability of a person to effectively respond to emotional experience. It was developed by Gross and John (2003). It consisted of 10 items. The construct has two subscales including reappraisal and suppression. The scale is based on 7-point Likert response format. Scores range from 10 to 70 as minimum and maximum. All the items are positively worded. The test-retest reliability of original scale is .79. The previous research in the European context confirmed that scale is valid and reliable to measure emotional regulation (Mauss et al., 2005).

Wong and Law Emotional Intelligence Scale was constructed to assess the emotional intelligence of a person. It was established by Wong and Law (2002). It consisted of 16 items with 4 subscales naming selfemotion appraisal, emotion appraisal of others, use of emotion, and regulation of emotion were included in the instrument. It is based on a 5-point Likert response format. Minimum and maximum scores are ranged from 16 to 80 . All the items are positively worded. The alpha reliability of the original instrument is .86 .

\section{Analyses}

Table 1: Psychometric properties

\begin{tabular}{|lccccccc|}
\hline Variables & $M$ & $S D$ & $\alpha$ & Potential & Actual & Skewness & Kurtosis \\
\hline 1. Emotional Intelligence & 49.46 & 8.24 & .78 & $16-80$ & $37-78$ & -.52 & -.42 \\
2. Emotional Regulation & 30.25 & 4.34 & .77 & $10-70$ & $49-47$ & -.14 & -.35 \\
3. Emotional Expressivity & 44.43 & 8.92 & .79 & $16-112$ & $32-69$ & -.34 & -.81 \\
4. Positive Emotional Expressivity & 15.11 & 3.15 & .80 & $8-56$ & $9-20$ & -.19 & -.14 \\
5. Negative Emotional Expressivity & 19.52 & 3.43 & 88 & $8-56$ & $9-26$ & -.82 & -.92 \\
\hline
\end{tabular}

The values of alpha reliability are satisfactory which confirms that all scales can be used effectively. Moreover, the normality data has confirmed appropriate level of normality.

Table 2: Pearson Correlation

\begin{tabular}{|c|c|c|c|c|c|}
\hline Variables & 1 & 2 & 3 & 4 & 5 \\
\hline 1. Emotional Intelligence & - & $.52 * * *$ & $.41 * *$ & $-.56 * * *$ & $-.71 * * *$ \\
\hline Emotional Regulation & & - & $.35 * *$ & $.22 * *$ & $-.25 * *$ \\
\hline Emotional Expressivity & & & - & $.19 *$ & $.18^{*}$ \\
\hline Positive Emotional Expressivity & & & & - & $-.52 * * *$ \\
\hline 5. Negative Emotional Expressivity & & & & & - \\
\hline
\end{tabular}


The correlations are desirable and in appropriate direction which are appropriate to make inferences as hypothesized.

\section{Discussion}

The study objective was to examine the relation among emotional intelligence, emotional regulation and emotional expressivity in employees. The reliability of the scales was ensured (Kline, 2005). The values of skewness and kurtosis for all scales indicate that data is normally distributed (Fitrianto, \& Chin, 2016). Previous study has found that when internet users are met with only text and no emoticons, they are unable to interpret the required sentiment, mood, or attention intent (Meng, \& Trudel, 2017). When emoticons are used in the same way, however, the recipient's view of the messages begins to shift dramatically. When opposite-meaning emoticons are used, the receiver's interpretations are radically different. Emoticons help people consider the intensity and direction of feeling, mood, and focus expression. These findings show that emoticons can be used for nonverbal communication (Trudel, Argo, \& Meng, 2015).

Emotions vary according to gender. Women are thought to receive an education that is biased toward emotional intelligence like anger, disgust etc., while men are thought to receive an education that is biased toward emotional intelligence like 79 such emotions such as grief, shame, weakness, and terror (Barrett, \& Bliss-Moreau, 2009; Hall, 2012). In comparison, women spend more time emotionally in touch with the relational environment (Shields, 2002) and are more concerned with preserving the optimistic sound of their and others' feelings in order to stop intimate relationships fracturing and to create fulfilling social networks (Fischer et al., 2018). Research evidence showed that women are more emotionally intelligent because of better social skills and high understanding of others emotions (Fischer, Kret, \& Broekens, 2018). Consequently, women are sensitive to expressing their emotions that's why they can decode others' emotions in a better way.

This study examined link between emotional intelligence, emotional regulation and emotional expressivity among employees in Pakistani context. Despite the impressive results, there are certain drawbacks that should be considered in future studies. The study's drawback is that it used a cross-sectional survey test design. While it has strong external validity, it has poor internal validity, making it impossible to establish causality paths. Longitudinal experiments should be undertaken in the future to capture human variations dynamically to develop a deeper understanding of the relationship between these factors. Another limitation of the study is the collection of data from only one culture due to time shortage and limited resources. The future research must be based on cross culture context and also include other demographic differences in a global context. One highlighted limitation was social desirability issues. The participants may likely repose in socially desirable behavior. This requires carefully handling of such social desirability related issues to make findings sound in future research.

\section{Conclusion}

The study investigated the association between emotional intelligence, emotion regulation and emotional expressivity among employees. The findings confirmed that these three emotions related constructs are correlated. Thus, improving even one of these factors related to emotions can be beneficial to improve other related factors of emotions because these are found interconnected through the present investigation.

\section{References}

Barrett L F \& Bliss-Moreau E. She's emotional. He's having a bad day: Attributional explanations for emotion stereotypes. Emotion. 2009; 9, 649-658. https://doi.org/10.1037/a0016821 PMID: 19803587

Brandão, T., Tavares, R., Schulz, M. S., \& Matos, P. M. (2016). Measuring emotion regulation and emotional expression in breast cancer patients: A systematic review. Clinical Psychology Review, 43, 114-127. 
Cardinosa-Queriones, E., Alvaro, J. K., \& Rosete, J. (2018). Knowledge in Emoticons of the ABEL Students. Southeast Asian Journal of Science and Technology, 3(1).

Colman A (2008). A Dictionary of Psychology (3 ed.). Oxford University Press.

El Bashir, H., Dekair, L., Mahmoud, Y., \& Ben-Omran, T. (2014). Neurodevelopmental and cognitive outcomes of classical homocystinuria: experience from Qatar. In JIMD Reports, Volume 21 (pp. 8995). Springer, Berlin, Heidelberg.

Fischer, A. H., Kret, M. E., \& Broekens, J. (2018). Gender differences in emotion perception and selfreported emotional intelligence: A test of the emotion sensitivity hypothesis. PloS one, 13(1).

Fitrianto, A., \& Chin, L. Y. (2016). Assessing normality for data with different sample sizes using SAS, Minitab and R. ARPN J Eng Appl Sci, 11, 10845-50.

Gross, J. J. (2014). "Emotion regulation: conceptual and empirical foundations. In ed J. J. Gross (Ed.). Handbook of Emotion Regulation (pp. 3-20). New York, NY: Guilford Press.

Gross, J. J., \& John, O. P. (1997). Revealing feelings: facets of emotional expressivity in self-reports, peer ratings, and behavior. Journal of Personality and Social Psychology, 72(2), 435.

Gross, J. J., \& John, O. P. (2003). Individual differences in two emotion regulation processes: Implications for affect, relationships, and well-being. Journal of Personality and Social Psychology, 85, 348-362.

Hall JA. Gender effects in decoding nonverbal cues. Psychological Bulletin. 1978; 85 (4), 84525. 19. Kret ME \& De Gelder B. A review on sex differences in processing emotional signals. Neuropsychologia. 2012; 50(7), 1211-1221. https://doi.org/10.1016/j.neuropsychologia.2011.12.022 PMID: 22245006

Jibril, T.A. and Abdullah, M.H., 2013. Relevance of emoticons in computer-mediated communication contexts: An overview. Asian Social Science, 9(4), p.201.

Kim, K. W., \& Park, D. H. (2018). Emoticon by Emotions: The Development of an Emoticon Recommendation System Based on Consumer Emotions. Journal of Intelligence and Information Systems, 24(1), 227-252.

Kline, T. J. (2005). Psychological testing: A Practical Approach to Design and Evaluation. Sage Publications.

Lin, H. and Qiu, L., 2012. Sharing emotion on Facebook: network size, density, and individual motivation. In CHI'12 Extended Abstracts on Human Factors in Computing Systems (pp. 2573-2578).

Lyons, A. (2018). Multimodal expression in written digital discourse: The case of kineticons. Journal of Pragmatics, 131, 18-29.

MANALAI, P., Manalai, G.G., MYINT, K., ATA, P. and HARACIC, A., 2012. Patients with psychotic disorders recognize facial emotional expression better in emoticons compared to in images. International Journal of Life Science \& Pharma Research, 2, pp.40-43.

Mauss, I. B., Levenson, R. W., McCarter, L., Wilhelm, F. H., \& Gross, J. J. (2005). The tie that binds? Coherence among emotion experience, behavior, and physiology. Emotion, 5(2), 175.

Meng, M. D., \& Trudel, R. (2017). Using emoticons to encourage students to recycle. The Journal of Environmental Education, 48(3), 196-204.

Ng, K. M., Wang, C., Zalaquett, C. P., \& Bodenhorn, N. (2007). A confirmatory factor analysis of the Wong and Law Emotional Intelligence Scale in a sample of international college students. International Journal for the Advancement of Counselling, 29(3-4), 173.

Peña-Sarrionandia, A., Mikolajczak, M., \& Gross, J. J. (2019). "Integrating emotion regulation and emotional intelligence traditions: A meta-analysis": Corrigendum. Frontiers in Psychology, 10, Article 2610. https://doi.org/10.3389/fpsyg.2019.02610

Riggio H.R. (2017) Emotional Expressiveness. In: Zeigler-Hill V., Shackelford T. (eds) Encyclopedia of Personality and Individual Differences. Springer, Cham. https://doi.org/10.1007/978-3-31928099-8_508-1

Sarkar, T., Shetty, N. and Humstoe, M.K., 2014. Emoticons \& emotions: Contextual interpretation in text messages \& consensus of meaning. Learning Curve, 3, pp.24-33.

Shields SA. Speaking from the heart: Gender and the social meaning of emotion. 2002; Cambridge, England: Cambridge University Press

Trudel, R., Argo, J. J., \& Meng, M. D. (2015). Trash or recycle? How product distortion leads to categorization error during disposal. Environment \& Behavior, 48(7), 966-985 
Vandergriff, I., 2013. Emotive communication online: A contextual analysis of computer-mediated communication (CMC) cues. Journal of Pragmatics, 51, pp.1-12.

Wojciechowski, J., Stolarski, M., \& Matthews, G. (2014). Emotional intelligence and mismatching expressive and verbal messages: a contribution to detection of deception. PloS one, 9(3), e92570. https://doi.org/10.1371/journal.pone.0092570

Zhang, J., \& Zheng, Y. (2017). How do academic stress and leisure activities influence college students' emotional well-being? A daily diary investigation. Journal of adolescence, 60, 114-118.
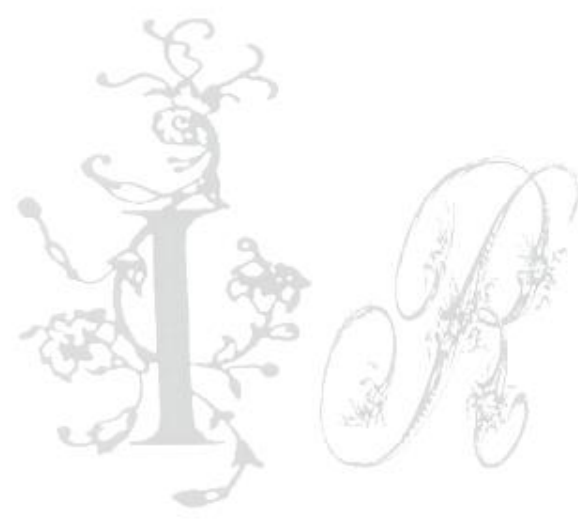